\title{
NECESSARY CONDITIONS FOR HYPONORMALITY OF TOEPLITZ OPERATORS ON THE FOCK SPACE
}

\author{
ANURADHA GUPTA AND SHIVAM KUMAR SINGH
}

Abstract. In this article, the necessary conditions for the hyponormality of Toeplitz operator $T_{\phi}$ with harmonic polynomial symbols $\phi$ on the Fock space are explored.

\section{INTRODUCTION}

Let $d A$ denote the ordinary Lebesgue area measure on the complex plane $\mathbb{C}$. Let the space $L^{2}(\mathbb{C}, d \mu)$ be the Hilbert space of all Lebesgue measurable, absolute square integrable functions on $\mathbb{C}$ with the norm given by

$$
\|f\|=\left(\int_{\mathbb{C}}|f(z)|^{2} d \mu(z)\right)^{1 / 2} \text { for each } f \in L^{2}(\mathbb{C}, d \mu)
$$

where the measure is given by $d \mu(z)=e^{-|z|^{2}} d A(z)$. The Fock space $\mathbb{F}^{2}$ consists of all entire functions in $L^{2}(\mathbb{C}, d \mu)$ and is a closed subspace of $L^{2}(\mathbb{C}, d \mu)$. The space $\mathbb{F}^{2}$ is a Hilbert space with the inner product inherited from $L^{2}(\mathbb{C}, d \mu)$ given by

$$
\langle f, g\rangle=\int_{\mathcal{C}} f(z) \overline{g(z)} d \mu(z) \text { where } f, g \in \mathbb{F}^{2} .
$$

For $n \geq 0$, let $e_{n}(z)=\frac{z^{n}}{\sqrt{\pi n !}}$, then the set $\left\{e_{n}\right\}_{n \geq 0}$ forms an orthonormal basis for $\mathbb{F}^{2}$ (see [12]). Let $P$ denote the orthogonal projection from the space $L^{2}(\mathbb{C}, d \mu)$ onto the space $\mathbb{F}^{2}$. Then, for any $f \in L^{2}(\mathbb{C}, d \mu)$ and $z \in \mathbb{C}$, it follows that

$$
P(f(z))=\left\langle P f(w), K_{z}(w)\right\rangle=\frac{1}{\pi} \int_{\mathbb{C}} f(w) e^{z \bar{w}} d \mu(w)
$$

where $K_{z}(w)=\overline{K(z, w)}=\frac{1}{\pi} e^{w \bar{z}}$ is the Fock Kernel. The Fock space plays a special role in quantum physics, harmonic analysis on the Heisenberg group, and partial differential equations.

Let the space $L^{\infty}(\mathbb{C})$ be the set of all essentially bounded Lebesgue measurable functions $f$ on the entire complex plane $\mathbb{C}$. The space $L^{\infty}(\mathbb{C})$ is the Banach space with the norm $\|f\|_{\infty}=\operatorname{ess} \sup \{|f(z)|: z \in \mathbb{C}\}$, for $f \in L^{\infty}(\mathbb{C})$. Then, for $\phi \in L^{\infty}(\mathbb{C})$, the multiplication operator denoted by $M_{\phi}$, is defined as the operator $M_{\phi}: L^{2}(\mathbb{C}, d \mu) \longrightarrow L^{2}(\mathbb{C}, d \mu)$ such that $M_{\phi}(f)=\phi f$ where $f \in L^{2}(\mathbb{C}, d \mu)$ and the Toeplitz operator is the operator $T_{\phi}$ defined on $\mathbb{F}^{2}$ so that $T_{\phi}(f)=P(\phi f)$ for

$M S C$ (2010): primary 47B35; secondary 47B20, 46E22.

Keywords: Toeplitz operator, Fock space, hyponormality.

Support of CSIR Research Grant to second author [F.No. 09/045(1405)/2015-EMR-I] for carrying out the research work is fully acknowledged. 
$f \in \mathbb{F}^{2}$. Consider the set $\mathcal{D}=\left\{\phi: \phi\right.$ is measurable and $\phi f \in L^{2}(\mathbb{C}, d \mu)$ for all $f \in$ $\left.\mathbb{F}^{2}\right\}$. Then, $L^{\infty}(\mathbb{C}) \subset \mathcal{D}$ and Toeplitz operator $T_{\phi}$ is also well defined for $\phi \in \mathcal{D}$. The study of Toeplitz operators gained voluminous importance due to its multidirectional applications. Toeplitz operators arise in many applications such as prediction theory, wavelet analysis and differential equations.

A bounded linear operator $S$ on a Hilbert space is said to be hyponormal if its self-commutator $\left[S^{\star}, S\right]=S^{\star} S-S S^{\star}$ is positive definite, where $S^{\star}$ is the adjoint of the operator $S$. In $[1,2]$, C. Cowen gave an elegant characterization of hyponormal Toeplitz operator on the Hardy space. Later on, Nakazi and Takahashi [7] also characterized the hyponormality of Toeplitz operators on Hardy space using a different approach given by Cowen. In the Bergman space, Sadaroui [10], Hwang and Lee [3-6], Phukon and Munmun [8,9] studied the hyponormality of Toeplitz operators. Motivated by the work of these authors, we have studied the hyponormality of Toeplitz operators $T_{\phi}$ on Fock space $\mathbb{F}^{2}$ with polynomial harmonic symbols $\phi$. We have obtained some necessary conditions for Toeplitz operators to be hyponormal on the Fock space.

\section{NECESSARY CONDITIONS FOR HYPONORMALITY}

Since the hyponormality of operators is translation invariant, we may assume that $f(0)=g(0)=0$ for $f, g \in \mathcal{D}$. The following proposition follows from the definition of Toeplitz operators.

Proposition 2.1. If $f, g \in \mathcal{D}$, then the following equations hold:

(1) $T_{f+g}=T_{f}+T_{g}$

(2) $T_{f}^{\star}=T_{\bar{f}}$

(3) $T_{\bar{f}} T g=T_{\bar{f} g}$ if $f$ or $g$ is analytic.

Lemma 2.2. If $P$ is an orthogonal projection on $\mathbb{F}^{2}$, then for any non-negative integers $s$ and $t$, we have

$$
P\left(\bar{z}^{t} z^{s}\right)= \begin{cases}\frac{s !}{(s-t) !} z^{s-t}, & \text { for } s \geq t \\ 0, & \text { otherwise }\end{cases}
$$

Proof. For non-negative integers $s$ and $t$ with $s \geq t$, using the measure $d \mu(z)$, we have

$$
\begin{aligned}
P\left(\bar{z}^{t} z^{s}\right) & =\left\langle P\left(\bar{w}^{t} w^{s}\right), K_{z}(w)\right\rangle=\frac{1}{\pi} \int_{\mathbb{C}} \bar{w}^{t} w^{s} e^{z \bar{w}} d \mu(w)=\frac{1}{\pi} \int_{\mathbb{C}} \bar{w}^{t} w^{s} e^{z \bar{w}-|w|^{2}} d A(w) \\
& =\frac{1}{\pi} \int_{\mathbb{C}} \bar{w}^{t} w^{s} \sum_{n=0}^{\infty} \frac{(z \bar{w})^{n}}{n !} e^{-|w|^{2}} d A(w) \\
& =\frac{1}{\pi} \sum_{n=0}^{\infty} \frac{z^{n}}{n !} \int_{\mathbb{C}} \bar{w}^{t} w^{s}(\bar{w})^{n} e^{-|w|^{2}} d A(w)
\end{aligned}
$$


The last step follows from the fact that the series is uniformly convergent. Now letting $w=r e^{i \theta}$ for $0 \leq \theta \leq 2 \pi$ and $0<r<\infty$, we have

$$
\begin{aligned}
P\left(\bar{z}^{t} z^{s}\right) & =\frac{1}{\pi} \sum_{n=0}^{\infty} \frac{z^{n}}{n !} \int_{r=0}^{\infty} \int_{\theta=0}^{2 \pi} r^{s+n+t+1} e^{i(s-n-t) \theta} e^{-r^{2}} d \theta d r \\
& =2 \frac{z^{s-t}}{(s-t) !} \int_{r=0}^{\infty} r^{2 s+1} e^{-r^{2}} d r \\
& =2 \frac{z^{s-t}}{(s-t) !} \int_{t=0}^{\infty} t^{s} e^{-t} \frac{d t}{2}\left(\text { for } t=r^{2}\right) \\
& =\frac{z^{s-t}}{(s-t) !} \Gamma(s+1),
\end{aligned}
$$

where $\Gamma$ denotes the Gamma function. Therefore, for $s \geq t$, we have

$$
P\left(\bar{z}^{t} z^{s}\right)=\frac{s !}{(s-t) !} z^{s-t}
$$

Similarly, for $s<t$, it follows that

$$
\begin{aligned}
P\left(\bar{z}^{t} z^{s}\right) & =\frac{1}{\pi} \sum_{n=0}^{\infty} \frac{z^{n}}{n !} \int_{r=0}^{\infty} \int_{\theta=0}^{2 \pi} r^{s+n+t+1} e^{i(s-n-t) \theta} e^{-r^{2}} d \theta d r \\
& =\frac{1}{\pi} \sum_{n=0}^{\infty} \frac{z^{n}}{n !}\left(\int_{r=0}^{\infty} r^{s+n+t+1} e^{-r^{2}} d r \int_{\theta=0}^{2 \pi} e^{i(s-n-t) \theta} d \theta\right)
\end{aligned}
$$

Now using the fact $\int_{\theta=0}^{2 \pi} e^{i m \theta} d \theta=\left\{\begin{array}{ll}2 \pi & \text { if } m=0 \\ 0 & \text { otherwise }\end{array}\right.$ and $n \neq s-t$ (because $\left.s-t<0\right)$, we get that $\int_{\theta=0}^{2 \pi} e^{i(s-n-t) \theta} d \theta=0$ for all $n \geq 0$. Hence, $P\left(\bar{z}^{t} z^{s}\right)=0$.

Thus, it follows that (2.1) holds.

For non-negative integers $s$ and $t$, from ([11], Lemma 2.1), it follows that

$$
\left\langle z^{s}, z^{t}\right\rangle= \begin{cases}\pi s ! & \text { if } s=t \\ 0 & \text { otherwise. }\end{cases}
$$

Toeplitz operators $T_{\phi}$ with symbols $\phi \in \mathcal{D}$ of the form $\phi(z)=z^{k}$, for some positive integers $k$, are always hyponormal on the Fock space. But with more general symbols, Toeplitz operators need not be hyponormal. In the following theorem, we present generalised necessary conditions for the hyponormality of Toeplitz operator $T_{\phi}$, with a polynomial harmonic symbol $\phi$ in $\mathcal{D}$.

Theorem 2.3. Let $\phi(z)=f(z)+\overline{g(z)}$, where $f(z)=\sum_{n=1}^{N} a_{n} z^{n}$ and $g(z)=$ $\sum_{n=1}^{N} a_{-n} z^{n} ; N \geq 2$ is an integer. If $T_{\phi}$ is hyponormal, then

(1) $\left|a_{1}\right|^{2}+\sum_{n=2}^{N}(n+1) !\left|a_{n}\right|^{2} \geq\left|a_{-1}\right|^{2}+\sum_{n=2}^{N}(n+1) !\left|a_{-n}\right|^{2}$. 
(2) $\sum_{n=1}^{N} n !\left|a_{n}\right|^{2} \geq \sum_{n=1}^{N} n !\left|a_{-n}\right|^{2}$.

(3) $\left(\sum_{n=1}^{N} n !\left(\left|a_{n}\right|^{2}-\left|a_{-n}\right|^{2}\right)\right)\left(\sum_{n=2}^{N}(n+1) !\left(\left|a_{n}\right|^{2}-\left|a_{-n}\right|^{2}\right)\right.$

$$
\left.+\left(\left|a_{1}\right|^{2}-\left|a_{-1}\right|^{2}\right)\right) \geq\left|\sum_{n=2}^{N} n !\left(a_{n} \overline{a_{n-1}}-a_{-n} \overline{a_{-(n-1)}}\right)\right|^{2} .
$$

Proof. Let the operator $T_{\phi}$ be hyponormal, then for complex numbers $c_{0}, c_{1}$, it follows that

$$
\left\langle\left(T_{\phi}^{\star} T_{\phi}-T_{\phi} T_{\phi}^{\star}\right)\left(c_{0}+c_{1} z\right),\left(c_{0}+c_{1} z\right)\right\rangle \geq 0
$$

where $\phi(z)=\sum_{n=1}^{N} a_{n} z^{n}+\sum_{n=1}^{N} \overline{a_{-n}} \bar{z}^{n}$.

Consider

$$
\begin{aligned}
& T_{\phi}\left(c_{0}+c_{1} z\right)=P\left(\phi\left(c_{0}+c_{1} z\right)\right) \\
= & P\left[c_{0}\left(\sum_{n=1}^{N} a_{n} z^{n}+\sum_{n=1}^{N} \overline{a_{-n}} \bar{z}^{n}\right)+c_{1}\left(\sum_{n=1}^{N} a_{n} z^{n+1}+\sum_{n=1}^{N} \overline{a_{-n}} \bar{z}^{n} z\right)\right] .
\end{aligned}
$$

Then, by using Lemma 2.2 , it follows that

$$
\begin{aligned}
T_{\phi}\left(c_{0}+c_{1} z\right) & =c_{0} \sum_{n=1}^{N} a_{n} z^{n}+c_{1}\left(\sum_{n=1}^{N} a_{n} z^{n+1}+\sum_{n=1}^{N} \overline{a_{-n}} P\left(\bar{z}^{n} z\right)\right) \\
& =c_{0} \sum_{n=1}^{N} a_{n} z^{n}+c_{1}\left(\sum_{n=1}^{N} a_{n} z^{n+1}+\overline{a_{-1}}\right)
\end{aligned}
$$

(since $P\left(\bar{z}^{n} z\right)=0$ for $\left.n>1\right)$. Therefore,

$$
\begin{aligned}
& \left\langle T_{\phi}^{\star} T_{\phi}\left(c_{0}+c_{1} z\right),\left(c_{0}+c_{1} z\right)\right\rangle=\left\langle T_{\phi}\left(c_{0}+c_{1} z\right), T_{\phi}\left(c_{0}+c_{1} z\right)\right\rangle \\
& =\left\langle c_{0} \sum_{n=1}^{N} a_{n} z^{n}+c_{1}\left(\overline{a_{-1}}+\sum_{n=1}^{N} a_{n} z^{n+1}\right), c_{0} \sum_{n=1}^{N} a_{n} z^{n}\right. \\
& \left.\quad+c_{1}\left(\overline{a_{-1}}+\sum_{n=1}^{N} a_{n} z^{n+1}\right)\right\rangle .
\end{aligned}
$$

Now from relation (2.2), it follows that

$$
\begin{aligned}
\left\langle T_{\phi}^{\star} T_{\phi}\left(c_{0}+c_{1} z\right),\left(c_{0}+c_{1} z\right)\right\rangle \\
=\left|c_{0}\right|^{2} \sum_{n=1}^{N}\left|a_{n}\right|^{2} \pi n !+\left|c_{1}\right|^{2}\left(\pi\left|a_{-1}\right|^{2}+\sum_{n=1}^{N}\left|a_{n}\right|^{2} \pi(n+1) !\right) \\
\quad+2 \operatorname{Re} c_{0} \overline{c_{1}}\left\langle\sum_{n=1}^{N} a_{n} z^{n}, \sum_{m=1}^{N} a_{m} z^{m+1}\right\rangle \\
=\pi\left\{\left|c_{0}\right|^{2} \sum_{n=1}^{N}\left|a_{n}\right|^{2} n !+\left|c_{1}\right|^{2}\left(\left|a_{-1}\right|^{2}+\sum_{n=1}^{N}\left|a_{n}\right|^{2}(n+1) !\right)\right. \\
\left.\quad+2 \operatorname{Re} c_{0} \overline{c_{1}} \sum_{n=2}^{N} a_{n} \overline{a_{n-1}} n !\right\} .
\end{aligned}
$$


Again, as $T_{\phi}^{\star}=T_{\bar{\phi}}$, by Lemma 2.2 , we have

$$
\begin{aligned}
& T_{\phi}^{\star}\left(c_{0}+c_{1} z\right)=P\left(\bar{\phi}\left(c_{0}+c_{1} z\right)\right) \\
= & P\left[c_{0}\left(\sum_{n=1}^{N} \overline{a_{n}} \bar{z}^{n}+\sum_{n=1}^{N} a_{-n} z^{n}\right)+c_{1}\left(\sum_{n=1}^{N} \overline{a_{n}} \bar{z}^{n} z+\sum_{n=1}^{N} a_{-n} z^{n+1}\right)\right] \\
= & c_{0} \sum_{n=1}^{N} a_{-n} z^{n}+c_{1}\left(\overline{a_{1}}+\sum_{n=1}^{N} a_{-n} z^{n+1}\right) .
\end{aligned}
$$

Thus, by relation $(2.2)$, we get

$$
\begin{aligned}
\langle & \left.T_{\phi} T_{\phi}^{\star}\left(c_{0}+c_{1} z\right),\left(c_{0}+c_{1} z\right)\right\rangle \\
= & \left\langle T_{\phi}^{\star}\left(c_{0}+c_{1} z\right), T_{\phi}^{\star}\left(c_{0}+c_{1} z\right)\right\rangle \\
= & \left\langle c_{0} \sum_{n=1}^{N} a_{-n} z^{n}+c_{1}\left(\overline{a_{1}}+\sum_{n=1}^{N} a_{-n} z^{n+1}\right), c_{0} \sum_{n=1}^{N} a_{-n} z^{n}\right. \\
& \left.+c_{1}\left(\overline{a_{1}}+\sum_{n=1}^{N} a_{-n} z^{n+1}\right)\right\rangle \\
= & \left|c_{0}\right|^{2} \sum_{n=1}^{N}\left|a_{-n}\right|^{2} \pi n !+\left|c_{1}\right|^{2}\left(\pi\left|a_{1}\right|^{2}+\sum_{n=1}^{N}\left|a_{-n}\right|^{2} \pi(n+1) !\right) \\
& +2 R e c_{0} \bar{c}_{1}\left\langle\sum_{n=1}^{N} a_{-n} z^{n}, \sum_{m=1}^{N} a_{-m} z^{m+1}\right\rangle \\
= & \pi\left\{\left|c_{0}\right|^{2} \sum_{n=1}^{N}\left|a_{-n}\right|^{2} n !+\left|c_{1}\right|^{2}\left(\left|a_{1}\right|^{2}+\sum_{n=1}^{N}\left|a_{-n}\right|^{2}(n+1) !\right)\right. \\
& \left.+2 \operatorname{Re} c_{0} \overline{c_{1}} \sum_{n=2}^{N} a_{-n} \overline{a_{-(n-1)}} n !\right\} .
\end{aligned}
$$

Therefore, by equations (2.4) and (2.5), it follows

$$
\begin{aligned}
& \left\langle\left(T_{\phi}^{\star} T_{\phi}-T_{\phi} T_{\phi}^{\star}\right)\left(c_{0}+c_{1} z\right),\left(c_{0}+c_{1} z\right)\right\rangle \\
= & \pi\left\{\left|c_{0}\right|^{2}\left(\sum_{n=1}^{N} n !\left(\left|a_{n}\right|^{2}-\left|a_{-n}\right|^{2}\right)\right)+\left|c_{1}\right|^{2}\left(\left|a_{-1}\right|^{2}-\left|a_{1}\right|^{2}\right)\right. \\
& +\left|c_{1}\right|^{2}\left(\sum_{n=1}^{N}(n+1) !\left(\left|a_{n}\right|^{2}-\left|a_{-n}\right|^{2}\right)\right) \\
& \left.+2 \operatorname{Re}\left(c_{0} \overline{c_{1}} \sum_{n=2}^{N} n !\left(a_{n} \overline{a_{n-1}}-a_{-n} \overline{a_{-(n-1)}}\right)\right)\right\} .
\end{aligned}
$$

Now from inequality (2.3), we get that

$$
\left|c_{0}\right|^{2}\left(\sum_{n=1}^{N} n !\left(\left|a_{n}\right|^{2}-\left|a_{-n}\right|^{2}\right)\right)
$$




$$
\begin{aligned}
& +\left|c_{1}\right|^{2}\left(\left|a_{1}\right|^{2}-\left|a_{-1}\right|^{2}+\sum_{n=2}^{N}(n+1) !\left(\left|a_{n}\right|^{2}-\left|a_{-n}\right|^{2}\right)\right) \\
& +2\left|c_{0} c_{1}\right|\left|\sum_{n=2}^{N} n !\left(a_{n} \overline{a_{n-1}}-a_{-n} \overline{a_{-(n-1)}}\right)\right| \geq 0 .
\end{aligned}
$$

Now the following three cases arise:

Case (1) If $c_{0}=0, c_{1} \neq 0$, then, by inequality (2.6), it follows that

$$
\left|a_{1}\right|^{2}-\left|a_{-1}\right|^{2}+\sum_{n=2}^{N}(n+1) !\left(\left|a_{n}\right|^{2}-\left|a_{-n}\right|^{2}\right) \geq 0
$$

or, equivalently,

$$
\left|a_{1}\right|^{2}+\sum_{n=2}^{N}(n+1) !\left|a_{n}\right|^{2} \geq\left|a_{-1}\right|^{2}+\sum_{n=2}^{N}(n+1) !\left|a_{-n}\right|^{2} .
$$

Case (2) If $c_{1}=0, c_{0} \neq 0$, then, by inequality (2.6), it follows that

$$
\sum_{n=1}^{N} n !\left(\left|a_{n}\right|^{2}-\left|a_{-n}\right|^{2}\right) \geq 0
$$

or, equivalently,

$$
\sum_{n=1}^{N} n !\left|a_{n}\right|^{2} \geq \sum_{n=1}^{N} n !\left|a_{-n}\right|^{2}
$$

Case (3) If $c_{0}, c_{1} \neq 0$, then, by inequality (2.6) it follows that

$$
\begin{aligned}
& \left|\frac{c_{0}}{c_{1}}\right|^{2} \sum_{n=1}^{N} n !\left(\left|a_{n}\right|^{2}-\left|a_{-n}\right|^{2}\right)+2\left|\frac{c_{0}}{c_{1}}\right|\left|\sum_{n=2}^{N} n !\left(a_{n} \overline{a_{n-1}}-a_{-n} \overline{a_{-(n-1)}}\right)\right| \\
& +\left(\sum_{n=2}^{N}(n+1) !\left(\left|a_{n}\right|^{2}-\left|a_{-n}\right|^{2}\right)+\left(\left|a_{1}\right|^{2}-\left|a_{-1}\right|^{2}\right)\right) \geq 0
\end{aligned}
$$

which is a quadratic polynomial in $\left|c_{0} / c_{1}\right|$ that only takes on non-negative values. But, if a quadratic polynomial $f(x)=a x^{2}+b x+c$ (for $a, b, c$ real and $a \geq 0$ ) takes on non negative values for all $x$, then it cannot have two distinct real roots. Hence, in such a case, the above equation has a non-positive discriminant and consequently we have,

$$
\begin{aligned}
& 4\left(\sum_{n=1}^{N} n !\left(\left|a_{n}\right|^{2}-\left|a_{-n}\right|^{2}\right)\right)\left(\sum_{n=2}^{N}(n+1) !\left(\left|a_{n}\right|^{2}-\left|a_{-n}\right|^{2}\right)+\left(\left|a_{1}\right|^{2}-\left|a_{-1}\right|^{2}\right)\right) \\
& \geq 4\left|\sum_{n=2}^{N} n !\left(a_{n} \overline{a_{n-1}}-a_{-n} \overline{a_{-(n-1)}}\right)\right|^{2}
\end{aligned}
$$

or, equivalently, we get condition (3) of Theorem 2.3.

The following examples show that the conditions in the above theorem are only necessary but not sufficient: 
Example 2.4. Let $\phi(z)=z+2 z^{2}+\bar{z}^{3}$, then it satisfies the conditions of Theorem 2.3. Then, by Lemma 2.2 , we have $T_{\phi}\left(z^{2}\right)=z^{3}+2 z^{4}$ and $T_{\phi}^{\star}\left(z^{2}\right)=z^{5}+2 z+4$. So, by relation (2.2) it follows that $\left\langle T_{\phi}^{\star} T_{\phi} z^{2}, z^{2}\right\rangle=102 \pi$ and $\left\langle T_{\phi} T_{\phi}^{\star}\left(z^{2}\right),\left(z^{2}\right)\right\rangle=$ $140 \pi$. Therefore, we get that $\left\langle\left(T_{\phi}^{\star} T_{\phi}-T_{\phi} T_{\phi}^{\star}\right)\left(z^{2}\right),\left(z^{2}\right)\right\rangle<0$ and thus $T_{\phi}$ is not hyponormal.

The following results are direct consequences of Theorem 2.3.

Corollary 2.5. Let $\phi(z)=f(z)+\overline{g(z)}$, where $f(z)=a_{m} z^{m}+a_{N} z^{N}$ and $g(z)=a_{-m} z^{m}+a_{-N} z^{N}$ for $1<m<N$. Let $T_{\phi}$ be hyponormal. Then,

(1) For $N \neq m+1$,

(a) $(m+1) !\left(\left|a_{m}\right|^{2}-\left|a_{-m}\right|^{2}\right) \geq(N+1) !\left(\left|a_{-N}\right|^{2}-\left|a_{N}\right|^{2}\right)$.

(b) $m !\left(\left|a_{m}\right|^{2}-\left|a_{-m}\right|^{2}\right) \geq N !\left(\left|a_{-N}\right|^{2}-\left|a_{N}\right|^{2}\right)$.

(2) For $N=m+1$,

(a) $(m+1) !\left(\left|a_{m}\right|^{2}-\left|a_{-m}\right|^{2}\right) \geq(m+2) !\left(\left|a_{-(m+1)}\right|^{2}-\left|a_{m+1}\right|^{2}\right)$.

(b) $m !\left(\left|a_{m}\right|^{2}-\left|a_{-m}\right|^{2}\right) \geq(m+1) !\left(\left|a_{-(m+1)}\right|^{2}-\left|a_{m+1}\right|^{2}\right)$.

(c) $\left\{\left(m !\left(\left|a_{m}\right|^{2}-\left|a_{-m}\right|^{2}\right)+(m+1) !\left(\left|a_{m+1}\right|^{2}-\left|a_{-(m+1)}\right|^{2}\right)\right.\right.$ $\left((m+1) !\left(\left|a_{m}\right|^{2}-\left|a_{-m}\right|^{2}\right)+(m+2) !\left(\left|a_{m+1}\right|^{2}-\left|a_{-(m+1)}\right|^{2}\right)\right\}$ $\geq\left[(m+1) !\left|a_{m+1} \overline{a_{m}}-a_{-(m+1)} \overline{a_{-m}}\right|\right]^{2}$.

Corollary 2.6. Let $\phi(z)=f(z)+\overline{g(z)}$, where $f(z)=a_{1} z+a_{N} z^{N}$ and $g(z)=$ $a_{-1} z+a_{-N} z^{N}$ for $N>1$. Let $T_{\phi}$ be hyponormal. Then,

(1) For $N>2$,

(a) $\left(\left|a_{1}\right|^{2}-\left|a_{-1}\right|^{2}\right) \geq N !\left(\left|a_{-N}\right|^{2}-\left|a_{N}\right|^{2}\right)$.

(b) $\left(\left|a_{1}\right|^{2}-\left|a_{-1}\right|^{2}\right) \geq(N+1) !\left(\left|a_{-N}\right|^{2}-\left|a_{N}\right|^{2}\right)$.

(2) For $N=2$,

$\left\{\left(\left|a_{1}\right|^{2}-\left|a_{-1}\right|^{2}+2\left(\left|a_{2}\right|^{2}-\left|a_{-2}\right|^{2}\right)\right)\left(\left|a_{1}\right|^{2}-\left|a_{-1}\right|^{2}+6\left(\left|a_{2}\right|^{2}-\left|a_{-2}\right|^{2}\right)\right)\right\}$

$\geq 4\left|a_{2} \overline{a_{1}}-a_{-2} \overline{a_{-1}}\right|^{2}$.

Again, the conditions in the above corollaries are only necessary but not sufficient which is shown by the following example:

Example 2.7. Let $\phi(z)=\sum_{i=1}^{4} a_{i} z^{i}+\sum_{j=1}^{4} a_{-j} \bar{z}^{j} \in \mathcal{D}$, where $a_{i}, a_{-j} \in \mathbb{C}$ for $1 \leq i, j \leq 4$. Now consider following four cases:

Case (1) $a_{2}=5, a_{4}=2 \sqrt{2}, a_{-2}=2, a_{-4}=3, a_{1}=a_{3}=a_{-1}=a_{-3}=0$. Then, $\phi(z)=5 z^{2}+2 \bar{z}^{2}+2 \sqrt{2} z^{4}+3 \bar{z}^{4}$ and it satisfies condition (1) of Corollary 2.5 , for $m=2$ and $N=4$. Then, by Lemma 2.2 , we have $T_{\phi}\left(z^{3}\right)=2 \sqrt{2} z^{7}+$ $5 z^{5}+12 z$ and so, by relation $(2.2)$, it follows that $\left\langle T_{\phi}^{\star} T_{\phi}\left(z^{3}\right),\left(z^{3}\right)\right\rangle=43464 \pi$. Also, we have $T_{\phi}^{\star}\left(z^{3}\right)=3 z^{7}+2 z^{5}+30 z$ and, again by relation $(2.2)$, we get that $\left\langle T_{\phi} T_{\phi}^{\star}\left(z^{3}\right),\left(z^{3}\right)\right\rangle=46740 \pi$. Consequently, it follows $\left\langle\left(T_{\phi}^{\star} T_{\phi}-T_{\phi} T_{\phi}^{\star}\right)\left(z^{3}\right),\left(z^{3}\right)\right\rangle$ $=(43464-46740) \pi=-3276 \pi<0$. Thus, $T_{\phi}$ is not hyponormal.

Case $(2) a_{2}=2, a_{-3}=1$ and $a_{i}=0$ for all $i \neq 2,-3$. Then, $\phi(z)=2 z^{2}+\bar{z}^{3}$ and it clearly satisfies condition (2) of Corollary 2.5. Then, by Lemma 2.2 it follows that $T_{\phi}\left(z^{4}\right)=2 z^{6}+24 z$ and $T_{\phi}^{\star}\left(z^{4}\right)=z^{7}+24 z^{2}$. Therefore, from relation $(2.2)$, it 
follows that $\left\langle T_{\phi}^{\star} T_{\phi}\left(z^{4}\right),\left(z^{4}\right)\right\rangle=3456 \pi$ and $\left\langle T_{\phi} T_{\phi}^{\star}\left(z^{4}\right),\left(z^{4}\right)\right\rangle=6192 \pi$. Therefore, we have $\left\langle\left(T_{\phi}^{\star} T_{\phi}-T_{\phi} T_{\phi}^{\star}\right)\left(z^{4}\right),\left(z^{4}\right)\right\rangle=-2736 \pi<0$. Hence, $T_{\phi}$ is not hyponormal.

Case $(3) a_{1}=5, a_{3}=2 \sqrt{2}, a_{-3}=3$ and $a_{i}=0$ for all $i \neq 1,3,-3$. Then, $\phi(z)=5 z+2 \sqrt{2} z^{3}+3 \bar{z}^{3}$, which satisfies condition (1) of Corollary 2.6, for $N=3$. Then, by Lemma 2.2 it follows that $T_{\phi}\left(z^{5}\right)=5 z^{6}+2 \sqrt{2} z^{8}+180 z^{2}$ and $T_{\phi}^{\star}\left(z^{5}\right)=25 z^{4}+120 \sqrt{2} z^{2}+3 z^{8}$. Therefore, from relation (2.2), it follows that $\left\langle T_{\phi}^{\star} T_{\phi}\left(z^{5}\right),\left(z^{5}\right)\right\rangle=405360 \pi$ and $\left\langle T_{\phi} T_{\phi}^{\star}\left(z^{5}\right),\left(z^{5}\right)\right\rangle=435480 \pi$. Consequently, $\left\langle\left(T_{\phi}^{\star} T_{\phi}-T_{\phi} T_{\phi}^{\star}\right)\left(z^{5}\right),\left(z^{5}\right)\right\rangle<0$. Thus, $T_{\phi}$ is not hyponormal.

Case (4) $a_{2}=1, a_{-1}=1, a_{-2}=2$ and $a_{i}=0$ for all $i \neq 2,-1,-2$. Then, $\phi(z)=$ $z^{2}+\bar{z}+2 \bar{z}^{2}$ which satisfies condition (2) of Corollary 2.6, with $a_{1}=0, a_{2}=1, a_{-1}=$ $1, a_{-2}=2$. Then, by Lemma 2.2 it follows that $T_{\phi}\left(z^{2}\right)=z^{4}+2 z+4$ and $T_{\phi}^{\star}\left(z^{2}\right)=$ $2 z^{4}+z^{3}+2$. Then, from relation (2.2), it follows that $\left\langle T_{\phi}^{\star} T_{\phi}\left(z^{2}\right),\left(z^{2}\right)\right\rangle=44 \pi$ and $\left\langle T_{\phi} T_{\phi}^{\star}\left(z^{2}\right),\left(z^{2}\right)\right\rangle=106 \pi$. Hence, $\left\langle\left(T_{\phi}^{\star} T_{\phi}-T_{\phi} T_{\phi}^{\star}\right)\left(z^{2}\right),\left(z^{2}\right)\right\rangle=44 \pi-106 \pi<0$. Thus, $T_{\phi}$ is not hyponormal.

Acknowledgement. The authors would like to express their sincere gratitude to the referees for their insightful and valuable comments and suggestions.

\section{REFERENCES}

[1] C. C. Cowen, Hyponormal and subnormal Toeplitz operators, in: J. B. Conway and B. B. Morrel (eds.), Surveys of some recent results in operator theory, vol. I, Pitman Research Notes in Mathematics Series 171, New York, John Wiley \& Sons, 1988, 155-167.

[2] C. C. Cowen, Hyponormality of Toeplitz operators, Proc. Amer. Math. Soc. 103 (1988), 809-812.

[3] I.S. Hwang, Hyponormal Toeplitz operators on the Bergman space, J. Korean Math. Soc. 42 (2005), 387-403.

[4] I.S. Hwang, Hyponormality of Toeplitz operators on the Bergman space, J. Korean Math. Soc. 45 (2008), 1027-1041.

[5] I.S. Hwang and J. Lee, Hyponormal Toeplitz operators on the Bergman space. II, Bull. Korean Math. Soc. 44 (2007), 517-522.

[6] J. Lee, Hyponormality of Toeplitz operators on the Bergman space, Kangweon-Kyungki Math. Jour. 15 (2007), 185-193.

[7] T. Nakazi and K. Takahashi, Hyponormal Toeplitz operators and extremal problems of Hardy spaces, Trans. Amer. Math. Soc. 338 (1993), 753-767.

[8] A. Phukon and M. Hazarika, On hyponormality of Toeplitz operators with polynomial and symmetric type symbols, Bull. Kor. Math. Soc. 48 (2011), 617-625.

[9] A. Phukon and M. Hazarika, Necessary conditions for hyponormality of Toeplitz operators on the Bergman space, Int. J. Math. Anal., Ruse 7 (2013), 485-490.

[10] H. Sadraoui, Hyponormality of Toeplitz operators and Composition operators, Thesis, Purdue University, 1992.

[11] S. Singh and A. Gupta, kth-order slant Toeplitz operators on the Fock space, Adv. Oper. Theory 2 (2017), 318-333.

[12] K. Zhu, Analysis on Fock spaces, Graduate Texts in Mathematics 263, New York, Springer, 2012. 
Anuradha Gupta, Department of Mathematics, Delhi College of Arts and Commerce, University of Delhi, Delhi-110023, India

e-mail: dishna2@yahoo.in

Shivam Kumar Singh, Department of Mathematics, University of Delhi, Delhi-110007, India e-mail: shivamkumarsingh14@gmail.com 
